\title{
Calculation Method of Short Term Flicker Severity Pst for Power System based on Atomic Decomposition and Real-Coded Quantum Evolutionary Algorithm
}

\author{
Hui Gao ${ }^{\mathrm{a},{ }^{*}}$, Qichao Song ${ }^{\mathrm{b}}$, Rui Zhang ${ }^{\mathrm{b}}$, and Jun Huang ${ }^{\mathrm{c}}$ \\ ${ }^{a}$ Department of Electrical Engineering and Automation, Heilongjiang Institute of Technology, Harbin, 158100, China \\ ${ }^{b}$ Department of Automation, Harbin University of Science and Technology, Harbin, 150080, China \\ ${ }^{c}$ Faculty of Geosciences and Environmental Engineering, Southwest Jiaotong University, Chengdu, 611756, China
}

\begin{abstract}
Short term flicker severity Pst is an important index to measure the power quality in the IEC standard, and the accurate calculation of Pst is the precondition to improve power quality. In order to improve the calculation accuracy of Pst, a novel method based on atomic decomposition and the real-coded quantum evolutionary algorithm is proposed to calculate the short time flicker severity Pst. Its core is that, firstly, on the basis of the Gabor complete atomic library, the real-coded quantum evolutionary algorithm is used to optimize the atomic parameters instead of using the matching pursuits algorithms, thereby improving search efficiency. Secondly, atomic decomposition technique based on real-coded quantum evolutionary algorithms is adopted to analyze harmonic components of the voltage fluctuation signal of power systems, which improves analysis ability. Finally, the proposed method is used to calculate Pst to improve calculation accuracy. Simulation experiment shows that based on the atomic decomposition and real-coded quantum evolutionary algorithm, the calculation results of the short time flicker severity Pst for power systems have higher precision compared with the results of other methods. This proves the validity and applicability of the proposed method.
\end{abstract}

Keywords: power quality; short term flicker severity; atomic decomposition; real-coded quantum evolutionary algorithm

(Submitted on February 5, 2018; Revised on March 15, 2018; Accepted on April 21, 2018)

(C) 2018 Totem Publisher, Inc. All rights reserved.

\section{Introduction}

With the development of the power market, most consumers have paid more and more attention to the quality of power, especially voltage [1]. The high-power load used in power grids causes rapid changes in power load. The power system has faced so much electrical pollution, such as power harmonics, voltage fluctuation, flicker and three-phase voltage imbalance. It also causes direct or potential harm to the safe operation of the power system. So, it is important to monitor power quality in real time and to take reasonable measures to improve the power quality based on the monitoring data.

Voltage fluctuation and flicker are important indicators of power quality [2]. Short term flicker severity Pst is a major comprehensive index that measures the quality of the power in the IEC standard, and the accurate calculation of Pst is the basis for monitoring power quality. In the past, FFT, which has some shortcomings such as spectrum leakage and fence effect, is used to calculate Pst.

This low calculation accuracy of Pst has a poor actual application effect. Literature [3,4] has put forward modifications of the FFT algorithm to overcome the shortcomings, but the interpolation cannot really fundamentally eliminate the influence of spectrum leakage and the fence effect. It is difficult for interpolation to improve the accuracy of calculation. At the same time, the IFFT algorithm is seriously affected by the noise $[5,6,7,8]$.

\footnotetext{
* Corresponding author.

E-mail address: gaohui_dl@sina.com
} 
In recent years, the atomic decomposition technique, which was presented by Mallat and Zhang to decompose the signal based on a complete atom library, has been widely used in the field of signal processing $[9,10]$. The atomic decomposition process uses essentially a series of related signals to approximate the original signals, and the original signal can be reconstructed in a smaller amount of computation, while parameters of the signal can be selected. The signal decomposition uses the matching pursuits algorithm [11]. That is to say, at each iteration, an atom is selected from the atomic library to match the residual component of the signal, and then the signal is expressed as a linear combination of the most matched atoms.

The atomic decomposition provides a new way to calculate the short time flicker severity Pst in power systems [12,13]. In this paper, a real-coded quantum evolutionary algorithm and an atomic decomposition based power system short time flicker severity Pst calculation method is proposed [14]. The atomic decomposition is applied to a power system short-term flicker severity Pst calculation. We take into account the existence of the MP algorithm, such as a large amount of computation and a search space for discretization $[15,16,17]$. In the discontinuous search space, it is impossible to find the global optimal solution. So, we use a real-coded quantum evolutionary algorithm to optimize the matching algorithm. Finally, simulation experiments show that the proposed method can greatly improve the calculation accuracy of the short time flicker severity Pst $[18,19,20]$.

\section{Atomic decomposition}

Suppose that $D=\left\{g_{k}, k=1,2, \ldots, K\right\}$ is an over-complete set in Hilbert space. If the element $g_{k}$ in $D$ is similar to the structure of signal $f$, the base of signal $f$ can be flexibly selected from $D$ by the characteristics of the signal itself. Because $D$ is complete and non-orthogonal, $g_{k}$ is no longer a true sense of the base, which is renamed to the atom, and then $D$ is called the atomic library, which can get a sparse decomposition of the signal $f$.

$$
\hat{f}=\sum_{k=0}^{N-1} c_{k} g_{k} \rightarrow f
$$

where $G$ is a constant coefficient.

In order to obtain a more concise and flexible sparse representation of the signal $f$, we need to select the atomic combination of the whole and local features of the best matching signal in a complete atomic library. That is, we need to minimize the energy of the error signal.

$$
\varepsilon=\|f-\hat{f}\| \rightarrow \min
$$

Gabor time-frequency atom has been widely used because of its good time-frequency resolution and time-frequency aggregation. The Gabor atom is made up of Gauss's function through expansion, translation and modulation. Its real expression is:

$$
g_{\gamma}(t)=\frac{K}{\sqrt{s}} g\left(\frac{t-\tau}{s}\right) \cos (\xi t+\varphi)
$$

where $g(t)=2^{1 / 4} e^{-\pi t^{2}}$ is the Gauss function; $K$ is the normalization factor; $S$ is the scale parameter and represents the span of the atom in the time domain. $u$ is the displacement factor, which represents the center position of the atom in the time domain envelope. $\xi$ is the frequency factor, which represents the center of the atom in the frequency domain envelope. $\phi$ is the phase factor, which represents the initial phase angle of the envelope atomic signal. From Formula (3) we can see that the $g_{\gamma}$ is uniquely determined by the time-frequency parameter $\gamma=(s, \tau, \xi, \phi)$.

The over complete atomic library obtained by Formula (3) is infinite, so it cannot be used in the actual situation. We need to discretize the time-frequency parameters of atoms in the atomic library to form a finite atomic library and ensure its completeness of the signal. The discretization method for atomic time-frequency parameters in the atomic library is: suppose that $\Delta \tau$ and $\Delta \xi$ are discrete intervals of time and frequency respectively, and both of them satisfy $\Delta \tau=\Delta \xi / 2 \pi<1$, then the time-frequency parameter $\gamma=(s, u, \xi, \varphi)$ discretization is:

$$
\gamma=\left(a^{j}, p a^{j} \Delta \tau, k a^{-j} \Delta \xi, i \Delta \varphi\right)
$$

The related parameters in Eq.(4) are set as Eq.(5). The atomic decomposition process adopts the MP algorithm. Suppose that $D$ is a discretized d over-complete Gabor atom library. For a signal $f$ of length $N$, the MP algorithm steps are as follows: 

into

First, atoms in the atomic library with the largest inner product of the signal are selected, which decomposes the signal

$$
\begin{aligned}
& \left\{\begin{array}{l}
a=2 \\
\Delta \tau=1 / 2 \\
\Delta \xi=\pi \\
\Delta \varphi=\pi / 6 \\
0 \leq j \leq \log _{2} N \\
0 \leq p \leq 2^{-j+1} N \\
0 \leq k<2^{j+1} \\
0 \leq i \leq 12
\end{array}\right. \\
& f(t)=\left\langle f(t), g_{\gamma(0)}(t)\right\rangle g_{\gamma(0)}(t)+R(t)
\end{aligned}
$$

where $R(t)$ is a signal and $f(t)$ is the residual signal after decomposition.

Then, the residual signals are decomposed by the same decomposition. It is selected from the atomic database in its most matching atom. By similar continuous iteration, when the accuracy requirements are met, the decomposition will stop. The signal can be expressed as

$$
f(t)=\sum_{k=0}^{m-1}\left\langle R^{k}(t), g_{\gamma(k)}(t)\right\rangle g_{\gamma(k)}(t)+R^{m}(t)
$$

where $R^{0}(t)=f(t) \cdot\left\|R^{m}(t)\right\|$ is a small constant and describes the size of the error after signal decomposition. So, we can use the $m$ atoms that have been selected to approximate the original signal. That is:

$$
f(t)=\sum_{k=0}^{m-1}\left\langle R^{k}(t), g_{\gamma(k)}(t)\right\rangle g_{\gamma(k)}(t)
$$

\section{Real-coded quantum evolutionary algorithm}

In Ref. [11,12], a real-coded chromosome, whose allele is composed of one component $x_{i}$ of variable vector $X$ and probability amplitudes $\left(\alpha_{i}, \beta_{i}\right)^{T}$ of one qubit, $i=1,2, \mathrm{~L}, n$, in RQEA is represented as:

$$
q=\left[\begin{array}{cccc}
x_{1} & x_{2} & \Lambda & x_{n} \\
\alpha_{1} & \alpha_{2} & \Lambda & \alpha_{n} \\
\beta_{1} & \beta_{2} & \Lambda & \beta_{n}
\end{array}\right]
$$

where $\mathrm{n}$ is the length of the chromosome and lies on the dimensions of variable vector $\mathrm{X}$.

The single-gene mutation is adopted to update the population at each iteration in RQEA. Assume that RQEA maintains a population $p(t)=\left\{p_{j}^{t}\right\}$ at the $t$-th iteration, where $j=1,2, \mathrm{~L}, N, N$ is the population size. Select the $i$-th gene $\left(x_{j i}^{t}, \alpha_{j i}^{t}, \beta_{j i}^{t}\right)^{T}$ of $p_{j}^{t}$ and update the value of $x_{j, i}^{t}$ using the Gaussian mutation, which is expressed as:

$$
x_{j, i}^{t+1, k}=x_{j i}^{t}+\left(x_{i, \max }-x_{i, \min }\right) N\left(0,\left(\sigma_{j, i}^{k}\right)^{2}\right)
$$

where $k \in\{\alpha, \beta\},\left(\sigma_{j, i}^{k}\right)^{2}$ denotes the Gaussian distribution variance, and its value is designed as:

$$
\left(\sigma_{j, i}^{k}\right)^{2}=\left\{\begin{array}{l}
\left|\alpha_{j, i}^{t}\right|^{2}, \quad k=\alpha \\
\left|\alpha_{j, i}^{t}\right|^{2} / 5, k=\beta
\end{array}\right.
$$


To avoid generating the infeasible solution, the value of $x_{j, i}^{t+1, k}$ is clipped according to Eq.(12). Until the value of $x_{j, i}^{t+1, k}$ lies in the feasible solution space, Eq.(5) has to be performed repeatedly.

$$
\left\{\begin{array}{l}
x_{j, i}^{t+1, k}=2 x_{i, \max }-x_{j, i}^{t+1, k}, x_{j, i}^{t+1, k}>x_{i, \max } \\
x_{j, i}^{t+1, k}=2 x_{i, \min }-x_{j, i}^{t+1, k}, x_{j, i}^{t+1, k}<x_{i, \min }
\end{array}\right.
$$

If the feasible solution derived from Eq.(10) (12) $\left.\left(x_{j, 1}^{t}, \mathrm{~L}, x_{j, i}^{t+1, k}, \mathrm{~L} x_{j, n}^{t}\right)^{T}\right)$ is superior to the feasible solution $\left(x_{j, 1}^{t}, \mathrm{~L}, x_{j, i}^{t}, \mathrm{~L} x_{j, n}^{t}\right)^{T}$, then the valid evolution is carried out, and $x_{j, i}^{t}=x_{j, i}^{t+1, k}, \alpha_{j, i}^{t+1}=\alpha_{j, i}^{t}, \beta_{j, i}^{t+1}=\beta_{j, i}^{t}$. Otherwise, the invalid evolution is done, the feasible solution $\left(x_{j, 1}^{t}, \mathrm{~L}, x_{j, i}^{t}, \mathrm{~L} x_{j, n}^{t}\right)^{T}$ is retained, and $\left(\alpha_{j i}^{t}, \beta_{j i}^{t}\right)^{T}$ is updated by quantum rotation gates as:

$$
\left[\begin{array}{c}
\alpha_{j, i}^{t+1} \\
\beta_{j, i}^{t+1}
\end{array}\right]=\left[\begin{array}{cc}
\cos \left(\Delta \theta_{j, i}^{t}\right) & -\sin \left(\Delta \theta_{j, i}^{t}\right) \\
\sin \left(\Delta \theta_{j, i}^{t}\right) & \cos \left(\Delta \theta_{j, i}^{t}\right)
\end{array}\right]\left[\begin{array}{c}
\alpha_{j, i}^{t+1} \\
\beta_{j, i}^{t+1}
\end{array}\right]
$$

where $\Delta \theta_{j, i}^{t}$ is the rotation angle, and the value of $\Delta \theta_{j, i}^{t}$ is designed as:

$$
\Delta \theta_{j, i}^{t}=\operatorname{sgn}\left(\alpha_{j, i}^{t} \beta_{j, i}^{t}\right) \theta_{0} \exp \left(-\frac{\left|\beta_{j, i}^{t}\right|}{\left|\alpha_{j, i}^{t}\right|+\gamma}\right)
$$

where $\operatorname{sgn}(\cdot)$ is the sign function that determines the direction of $\Delta \theta_{j, i}^{t}$ and $\theta_{0}$ is the initial rotation angle; $\gamma$ is evolutionary scale. $\theta_{0}, \gamma$ and $\left(\alpha_{j, i}^{t} \beta_{j, i}^{t}\right)^{T}$ decide the size of $\Delta \theta_{j, i}^{t}$ together and control the convergence rate of the algorithms.

From Eq. (13) and (14), we can see that, with an increase of iterations $t$, the value of $\left|\alpha_{j, i}^{t}\right|$ decreases gradually, and then 'Fine search' in the neighborhood of current solution is carried out. In reverse, the value of $\left|\beta_{j, i}^{t}\right|$ increases gradually, and then 'Coarse search' in the whole search space is realized. Based on 'Fine search' in the local search space and 'Coarse search' in the global search space, NRQEA can treat the balance between exploration and exploitation, which is the origin of the complementary mutation operator. In the case of $\left(\alpha_{j, i}^{t} \beta_{j, i}^{t}\right)^{T}$ in the Ith quadrant, Figure 1 demonstrates the principle of the complementary mutation operator. Let the number of "Fine search" and "Coarse search" for every individual be $m_{1}$ and $m_{2}$, respectively, and $m_{1}>m_{2}$.

In RQEA, discrete crossover is performed at period $\tau_{\mathrm{c}}$ to enhance the intercourse among the individuals and make use of the better gene obtained.

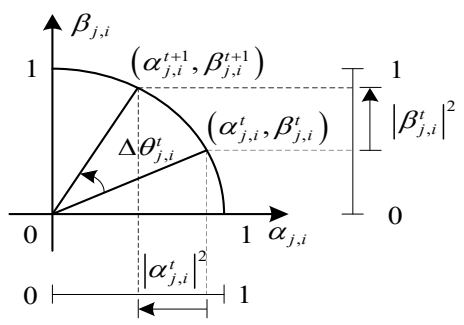

Figure 1. Principle of complementary mutation operator

The process of discrete crossover operator can be explained as follows: firstly, select $k$ excellent individuals from a population by the order of fitness value, and $k<N$. Then, for each excellent individual $p_{u}^{t}, u=1, \mathrm{~L}, k$, select another individual $p_{v}^{t}, v=1, \mathrm{~L}, N, v \neq u$, at random from the population and let $p_{u}^{t}$ and $p_{v}^{t}$ as parents. Exchange every corresponding gene by $1 / 2$ probability and generate the new individual $c^{t}$, which can be described as: 


$$
\left(\begin{array}{lll}
x_{c, i}^{t} & \alpha_{c, i}^{t} & \beta_{c, i}^{t}
\end{array}\right)^{T}=\left\{\begin{array}{lll}
\left(\begin{array}{lll}
x_{u, i}^{t} & \alpha_{u, i}^{t} & \beta_{u, i}^{t}
\end{array}\right)^{T}, & r<0.5 \\
\left(\begin{array}{lll}
x_{v, i}^{t} & \alpha_{v, i}^{t} & \beta_{v, i}^{t}
\end{array}\right)^{T}, & r \geq 0.5
\end{array}\right.
$$

where $\left(\begin{array}{lll}x_{c, i}^{t} & \alpha_{c, i}^{t} & \beta_{c, i}^{t}\end{array}\right)^{T},\left(\begin{array}{lll}x_{u, i}^{t} & \alpha_{u, i}^{t} & \beta_{u, i}^{t}\end{array}\right)^{T}$ and $\left(\begin{array}{lll}x_{v, i}^{t} & \alpha_{v, i}^{t} & \beta_{v, i}^{t}\end{array}\right)^{T}$ are the $i$ th gene of $p_{u}^{t}, p_{v}^{t}$ and $c^{t}$, respectively, and $r$ denotes a pseudo-random number selected with a uniform distribution over $(0,1)$.

RQEA puts in practice discrete crossover for each excellent individual selected $m_{3}$ times. If the fitness value of $c^{t}$ is superior to that of $p_{u}^{t}$, then $p_{u}^{t}$ is set to $c^{t}$. Otherwise, $p_{u}^{t}$ is retained. It is worthwhile to mention that, when the real-valued variables in Eq.(10) are strongly correlated, discrete crossover operator would play an important role on preventing algorithms from being trapped in the local optima.

\section{Calculation process and steps of the short time flicker severity Pst}

The calculation method of IEC definition of the short time flicker severity $P_{s t}$ is that, firstly, for the voltage fluctuation caused by the random variation of the load, the instantaneous visual sense of $S(t)$ is sampled at equal intervals within a long enough time of observation $T$ (at least 10min). Secondly, we classify the $S(t)$ data, count the probability distribution of each level, and then obtain the probability distribution function (CPF) by the probability of each level of data distribution. Finally, according to $\mathrm{CPF}$, level the statistical evaluation of flicker, that is, calculate flash $P_{s t}$. It can be seen that the instantaneous visual sensation directly reflects the influence of the voltage fluctuation on the human visual perception, which is the key point of the calculation. We can define the instantaneous value of the visual sense of the discrete $s(t)$ curve on the value of $p_{t}$ for the instantaneous flicker. According to the calculation method of the short time flicker severity defined by IEC, the calculation steps can be described as:

Step1: Sample the continuous voltage signal $u(t)$ to the discrete voltage signal $u(n)$. Make each half cycle of discrete signals $u(n)$ calculate a voltage RMS voltage to the RMS value of the sequence $U(n)$. The root mean square value curve of discrete voltage can be formed.

Step2: The voltage root mean square value sequence $U(n)$ is divided into equal intervals by time $\tau$, and the root mean square value sequence of the voltage is divided into $U_{i}(n), i=1, \mathrm{~L}, N . N$ is the total number divided into the observation time $T$, and $N=T / \tau$. Then, perform FFT analysis of the root mean square value sequence in each division, and then we can discrete spectral sequences $U_{f_{m}}(m), m=1, \mathrm{~L}, M . M$ is the upper limit of the frequency spectrum. We then get the corresponding frequency $f_{m}$, which is the sine voltage RMS curve of the peak value. The frequency of voltage fluctuation value $\Delta U_{f_{m}}(m)$ is $f_{m}$.

$$
\begin{gathered}
U_{f m}(m)=\operatorname{FFT}\left(U_{i}(n)\right) \\
\Delta U_{f_{m}}(m)=2\left|U_{f_{m}}(m)\right|
\end{gathered}
$$

Step3: The instantaneous flicker value $p_{i}$ of the $i$ voltage RMS sequence dividing $U_{i}(n)$ is equal to the sum of the corresponding instantaneous flicker values of each frequency $f_{m}$ on the corresponding frequency spectrum, that is:

$$
p_{i}=\sum_{m=1}^{M}\left[\frac{2\left|U_{f_{m}}(m)\right|}{d u_{m}}\right]^{2}, i=1, \Lambda, N
$$

where $d u_{m}$ is the sine voltage fluctuation value whose frequency is $f_{m}$ at the unit instantaneous flicker value. 
Step4: The instantaneous flicker value $p_{i}$ is divided into $L$ level by dividing the observation time $T$, because $p_{i}$ is equal interval data, the probability of data distribution in the class can be expressed by the frequency of the corresponding rank data $p(I), l=1, \mathrm{~L}, L$, as follows:

$$
p(l)=N_{l} / N
$$

where $N_{l}$ is the number of data distribution in class.

Step5: According to the probability of data distribution $P(l)$ a histPPogram can be obtained, and then form CPF. The CPF obtained by the instantaneous flicker value $P_{i}$ reflects the percentage of the instantaneous flicker value T over a certain limit of time to the observation time $P_{i}$. For the instantaneous flicker value CPF curve of the random variation load, the short time flicker severity $P_{s t}$ is calculated by the 5 prescribed values, they are as follows:

$P_{s t}=\left(0.0314 P_{0.1}+0.0525 P_{1}+0.0657 P_{3}+0.28 P_{10}+0.08 P_{50}\right)^{0.5}$ where $P_{0.1}, P_{1}, P_{3}$ and $P_{50}$ are the perceived unit value, which are instantaneous flicker value $P_{\mathrm{i}}$ over $0.1 \%, 1 \%, 3 \%, 10 \%$ and $50 \%$ time during observation time $T$ over $0.1 \%, 3 \%, 10 \%$ and times separately.

\section{Simulation experiment}

Through a large number of tests, IEC get the instantaneous flicker value of the unit sine wave voltage, rectangular wave voltage and voltage fluctuation value $\Delta V(\%$. In this paper, simulation test is done based on the IEC standard data, the fluctuation voltage is sampled at equal interval, and the sampling interval is $\Delta \mathrm{t}=10 \mathrm{~ms}$, that is, sampling frequency $f_{s}=1 / \Delta t=100 \mathrm{t}$, sampling length is $1 \mathrm{~s}$. As the fluctuation voltage waveform, voltage fluctuation value and the rate of determination are fixed, the calculated $P_{s i}$ fixed at 0.7144 . In order to compare the effectiveness of the method, the IFFT method and RQEA-Atom method are used to analyze the frequency spectrum. And the relationship between sine wave voltage of frequency and corresponding frequency is obtained by fitting the instantaneous flicker value in trained BP neural network.

Simulation test is carried out on the IEC standard to provide sinusoidal wave voltage and rectangular wave voltage at different frequencies. Table 1 and Table 2 were both compared with the experimental results. Due to limited space, Figure 2 and Figure 3 show the decomposing process of the sine wave and rectangular envelope signal with frequency of $8.8 \mathrm{H}$ using RQEA-Atom.

Table 1. Comparison of experimental results for sin wave

\begin{tabular}{|c|c|c|c|c|c|c|c|}
\hline \multirow{2}{*}{$f / \mathrm{Hz}$} & \multirow{2}{*}{$\Delta u / \%$} & \multicolumn{2}{|c|}{ FFT } & \multicolumn{2}{c|}{ IFFT } & \multicolumn{2}{c|}{ RQEA-Atom } \\
\cline { 3 - 8 } & & $P_{s t}$ & Error/\% & $P_{s t}$ & Error/\% & $P_{s t}$ & Error/\% \\
\hline 4.0 & 0.500 & 0.7132 & -0.1120 & 0.7144 & 0.0560 & $7.13975 \mathrm{e}-1$ & $-3.50 \mathrm{e}-3$ \\
\hline 4.5 & 0.445 & 0.7067 & -1.0224 & 0.7156 & 0.2240 & $7.14031 \mathrm{e}-1$ & $4.34 \mathrm{e}-3$ \\
\hline 5.0 & 0.398 & 0.6638 & -7.0308 & 0.7129 & -0.1540 & $7.13968 \mathrm{e}-1$ & $-4.48 \mathrm{e}-3$ \\
\hline 5.5 & 0.360 & 0.5952 & -16.6386 & 0.7129 & -0.1540 & $7.14053 \mathrm{e}-1$ & $7.42 \mathrm{e}-3$ \\
\hline 6.0 & 0.328 & 0.5050 & -29.2717 & 0.7145 & 0.0700 & $7.14025 \mathrm{e}-1$ & $3.50 \mathrm{e}-3$ \\
\hline 6.5 & 0.300 & 0.5087 & -28.7535 & 0.7143 & 0.0420 & $7.13962 \mathrm{e}-1$ & $-5.32 \mathrm{e}-3$ \\
\hline 7.0 & 0.280 & 0.6020 & -15.6862 & 0.7151 & 0.1540 & $7.13946 \mathrm{e}-1$ & $-7.56 \mathrm{e}-3$ \\
\hline 7.5 & 0.266 & 0.6698 & -6.1904 & 0.7150 & 0.1400 & $7.14037 \mathrm{e}-1$ & $5.18 \mathrm{e}-3$ \\
\hline 8.0 & 0.256 & 0.7042 & -1.3725 & 0.7113 & -0.3781 & $7.14042 \mathrm{e}-1$ & $5.88 \mathrm{e}-3$ \\
\hline 8.8 & 0.250 & 0.6997 & -2.0028 & 0.7128 & -0.1680 & $7.13969 \mathrm{e}-1$ & $-4.34 \mathrm{e}-3$ \\
\hline 9.5 & 0.254 & 0.6220 & -12.8851 & 0.7097 & -0.6022 & $7.14051 \mathrm{e}-1$ & $7.14 \mathrm{e}-3$ \\
\hline 10.0 & 0.262 & 0.5429 & -23.9635 & 0.7154 & 0.1960 & $7.13964 \mathrm{e}-1$ & $-5.04 \mathrm{e}-3$ \\
\hline 10.5 & 0.270 & 0.4698 & -34.2016 & 0.7138 & -0.0280 & $7.13953 \mathrm{e}-1$ & $-6.58 \mathrm{e}-3$ \\
\hline 11.0 & 0.282 & 0.5693 & -20.2661 & 0.7149 & 0.1260 & $7.14041 \mathrm{e}-1$ & $5.74 \mathrm{e}-3$ \\
\hline 11.5 & 0.296 & 0.6465 & -9.4537 & 0.7141 & 0.0140 & $7.14015 \mathrm{e}-1$ & $2.10 \mathrm{e}-3$ \\
\hline 12.0 & 0.312 & 0.6957 & -2.5630 & 0.7134 & -0.0840 & $7.14065 \mathrm{e}-1$ & $9.10 \mathrm{e}-3$ \\
\hline 13.0 & 0.348 & 0.6973 & -2.3389 & 0.7133 & -0.0980 & $7.13951 \mathrm{e}-1$ & $-6.86 \mathrm{e}-3$ \\
\hline 14.0 & 0.388 & 0.5739 & -19.6218 & 0.7148 & 0.1120 & $7.14019 \mathrm{e}-1$ & $2.66 \mathrm{e}-3$ \\
\hline 15.0 & 0.432 & 0.5372 & -24.7619 & 0.7138 & -0.0280 & $7.13989 \mathrm{e}-1$ & $-1.54 \mathrm{e}-3$ \\
\hline 16.0 & 0.480 & 0.6828 & -4.3697 & 0.7136 & -0.0560 & $7.13954 \mathrm{e}-1$ & $-6.44 \mathrm{e}-3$ \\
\hline 17.0 & 0.537 & 0.7067 & -1.0224 & 0.7137 & -0.0420 & $7.14022 \mathrm{e}-1$ & $3.08 \mathrm{e}-3$ \\
\hline 18.0 & 0.584 & 0.6015 & -15.7563 & 0.7144 & 0.0560 & $7.14041 \mathrm{e}-1$ & $5.74 \mathrm{e}-3$ \\
\hline 19.0 & 0.640 & 0.5043 & -29.3697 & 0.7131 & -0.1260 & $7.13976 \mathrm{e}-1$ & $-3.36 \mathrm{e}-3$ \\
\hline 20.0 & 0.700 & 0.6669 & -6.5966 & 0.7143 & 0.0420 & $7.13923 \mathrm{e}-1$ & $-1.07 \mathrm{e}-2$ \\
\hline
\end{tabular}


Table 2. Comparison of experimental results for square wave

\begin{tabular}{|c|c|c|c|c|c|c|c|}
\hline \multirow{2}{*}{$f / \mathrm{Hz}$} & \multirow{2}{*}{$\Delta u / \%$} & \multicolumn{2}{|c|}{ FFT } & \multicolumn{2}{c|}{ IFFT } & \multicolumn{2}{c|}{ RQEA-Atom } \\
\cline { 3 - 8 } & & $P_{s t}$ & Error/\% & $P_{s t}$ & Error/\% & $P_{s t}$ & Error/\% \\
\hline 4.0 & 0.333 & 0.6069 & -15.0000 & 0.6988 & -2.1288 & $7.1409 \mathrm{e}-1$ & 0.0126 \\
\hline 4.5 & 0.316 & 0.6832 & -4.3137 & 0.7008 & -1.8487 & $7.1394 \mathrm{e}-1$ & -0.0084 \\
\hline 5.0 & 0.293 & 0.6338 & -11.2324 & 0.7042 & -1.3725 & $7.1416 \mathrm{e}-1$ & 0.0224 \\
\hline 5.5 & 0.269 & 0.5662 & -20.7002 & 0.6966 & -2.4369 & $7.1445 \mathrm{e}-1$ & 0.0630 \\
\hline 6.0 & 0.249 & 0.5007 & -29.8739 & 0.7029 & -1.5546 & $7.1397 \mathrm{e}-1$ & -0.0042 \\
\hline 6.5 & 0.231 & 0.4991 & -30.0980 & 0.7069 & -0.9943 & $7.1383 \mathrm{e}-1$ & -0.0238 \\
\hline 7.0 & 0.217 & 0.6002 & -15.9383 & 0.7110 & -0.4201 & $7.1401 \mathrm{e}-1$ & 0.0014 \\
\hline 7.5 & 0.207 & 0.6653 & -6.8207 & 0.7126 & -0.1960 & $7.1453 \mathrm{e}-1$ & 0.0742 \\
\hline 8.0 & 0.201 & 0.7044 & -1.3445 & 0.7146 & 0.0840 & $7.1383 \mathrm{e}-1$ & -0.0238 \\
\hline 8.8 & 0.199 & 0.7090 & -0.7002 & 0.7224 & 1.1764 & $7.1457 \mathrm{e}-1$ & 0.0798 \\
\hline 9.5 & 0.200 & 0.6242 & -12.5770 & 0.7112 & -0.3921 & $7.1369 \mathrm{e}-1$ & -0.0434 \\
\hline 10.0 & 0.205 & 0.5586 & -21.7647 & 0.7175 & 0.4901 & $7.1388 \mathrm{e}-1$ & -0.0168 \\
\hline 10.5 & 0.213 & 0.4709 & -34.0476 & 0.7170 & 0.4201 & $7.1485 \mathrm{e}-1$ & 0.1190 \\
\hline 11.0 & 0.223 & 0.5718 & -19.9159 & 0.7197 & 0.7983 & $7.1385 \mathrm{e}-1$ & -0.0210 \\
\hline 11.5 & 0.234 & 0.6504 & -8.9075 & 0.7191 & 0.7142 & $7.1405 \mathrm{e}-1$ & 0.0070 \\
\hline 12.0 & 0.246 & 0.6989 & -2.1148 & 0.7168 & 0.3921 & $7.1354 \mathrm{e}-1$ & -0.0644 \\
\hline 13.0 & 0.275 & 0.7026 & -1.5966 & 0.7185 & 0.6302 & $7.1373 \mathrm{e}-1$ & -0.0378 \\
\hline 14.0 & 0.308 & 0.5815 & -18.5574 & 0.7227 & 1.2184 & $7.1389 \mathrm{e}-1$ & -0.0154 \\
\hline 15.0 & 0.344 & 0.5406 & -24.2857 & 0.7212 & 1.0084 & $7.1419 \mathrm{e}-1$ & 0.0266 \\
\hline 16.0 & 0.380 & 0.6893 & -3.4593 & 0.7201 & 0.8543 & $7.1413 \mathrm{e}-1$ & 0.0182 \\
\hline 17.0 & 0.333 & 0.7154 & 0.1960 & 0.7224 & 1.1764 & $7.1440 \mathrm{e}-1$ & 0.0560 \\
\hline 18.0 & 0.316 & 0.6065 & -15.0560 & 0.7186 & 0.6442 & $7.1394 \mathrm{e}-1$ & -0.0084 \\
\hline 19.0 & 0.293 & 0.5049 & -29.2857 & 0.7176 & 0.5042 & $7.1407 \mathrm{e}-1$ & 0.0098 \\
\hline 20.0 & 0.269 & 0.6807 & -4.6638 & 0.7291 & 2.1148 & $7.1340 \mathrm{e}-1$ & -0.0840 \\
\hline & & & & & & \\
\hline
\end{tabular}

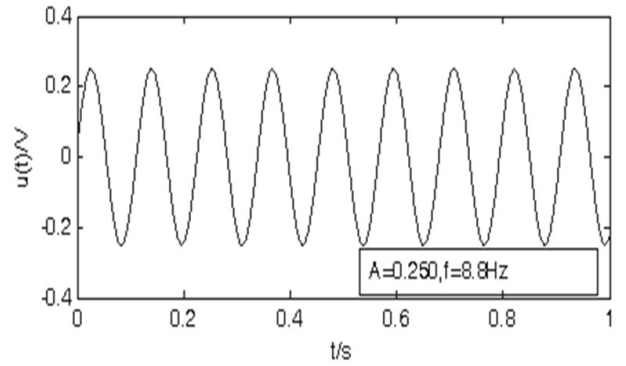

(a) The envelops signal

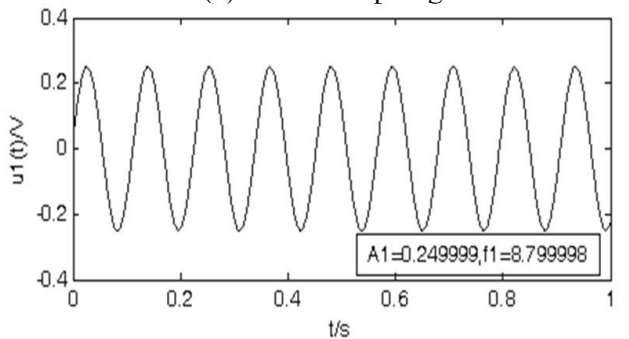

(b) The 1th decomposition of atoms

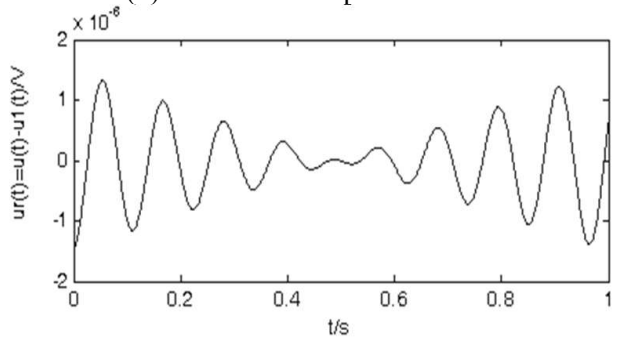

(c) The 1 th decomposition residual signal

Figure 2. $8.8 \mathrm{~Hz}$ sine wave signal decomposition process 
It can be seen from Table 1 and Table 2 that the proposed method for the sinusoidal fluctuation voltage and rectangular wave voltage has high accuracy, obviously better than FFT method and IFFT method, On the one hand, the calculated $P_{s i}$ by FFT method appears larger vibration, the calculated $P_{s i}$ by IFFT method is not stable enough. However, the calculated $P_{s i}$ by the method in this paper is very stable. On the other hand, the FFT method has a very poor accuracy in calculating $P_{s i}$. The IFFT method has a greater degree of improvement compared with the FFT method. However, the method in this paper has better accuracy than the FFT and the IFFT. Obviously, the proposed method is not only very precise, but also very stable.

From Figure 2, we can see that the $8.8 \mathrm{~Hz}$ sine wave envelope signal was decomposed by the RQEA-Atom, and the amplitude and frequency of the envelope signal are $0.249999 \mathrm{~V}$ and $8.799998 \mathrm{~Hz}$ respectively. They are close to the actual values with a very high precision. Figure 2 (c) depicts the error in reconstructing the envelope signal using the first decomposition results. It can be seen that the method is able to reconstruct the sine wave signal with high precision.

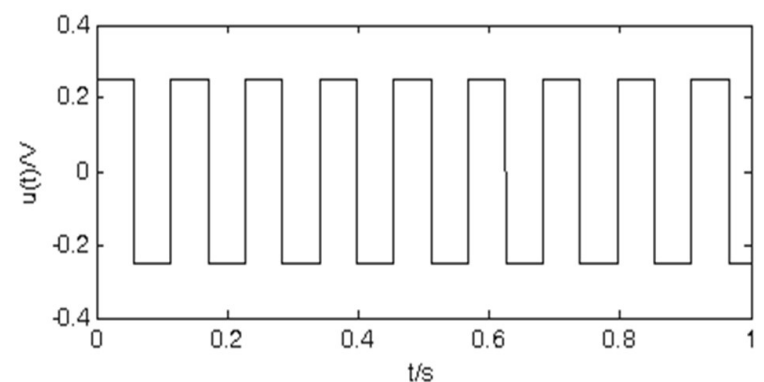

(a) The envelops signal

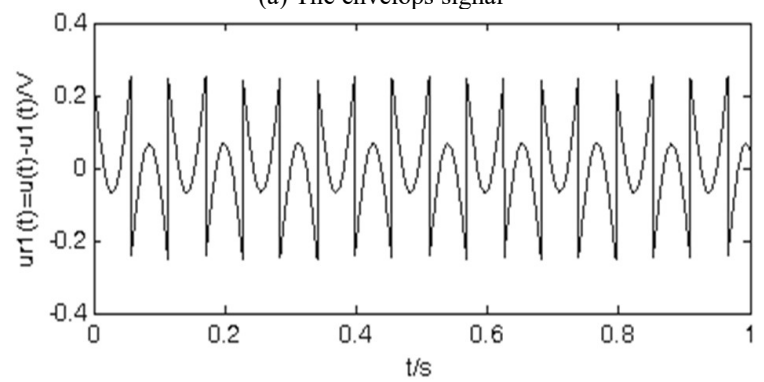

(c) The 1th decomposition residual signal

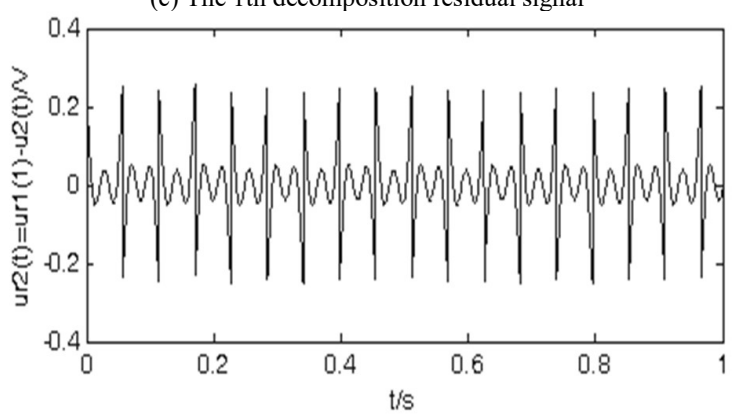

(e) The 2th decomposition residual signal

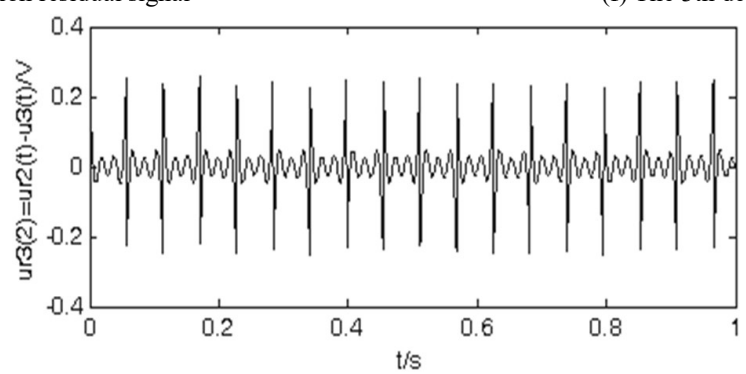

(g) The 3 th decomposition residual signal

Figure 3. describes the process of using RQEA-Atom to reconstruct the envelope of the rectangular wave.

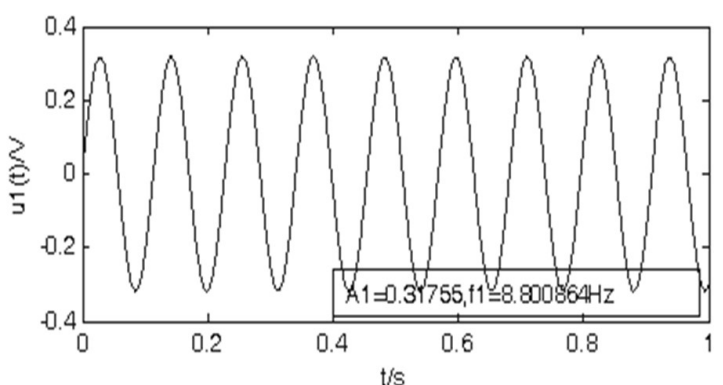

(b) The 1th decomposition of atoms

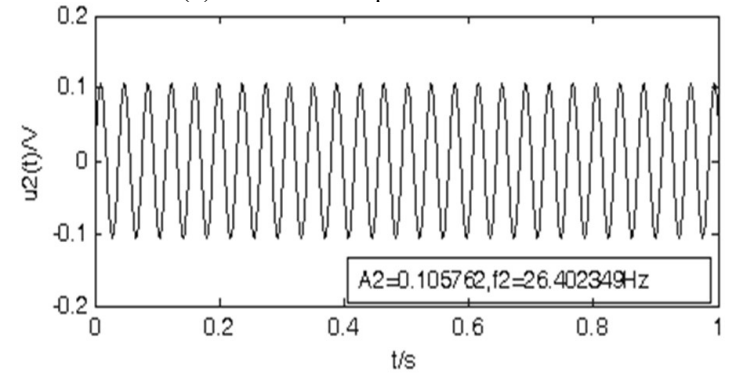

(d) The 2th decomposition of atoms

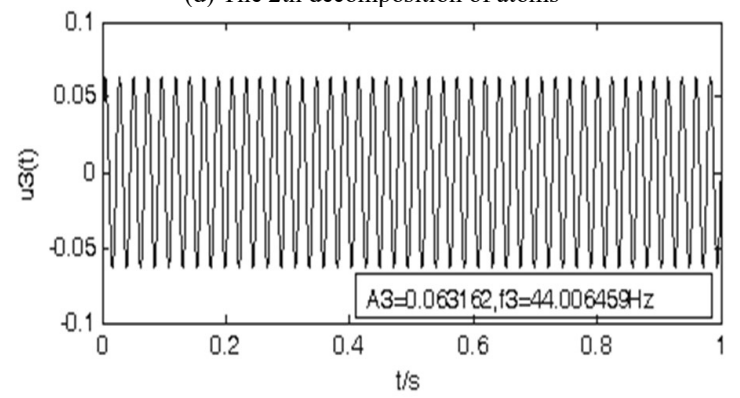

(f) The 3th decomposition of atoms 
From Figure 3 (b), it can be seen that the fundamental frequency of the envelope extracted by the first atomic decomposition is $0.317558 \mathrm{~V}$ and the frequency is 8.800864 . They are close to the theoretical value of $0.318309 \mathrm{~V}$ and $8.8 \mathrm{~Hz}$. From Figure 3 (d) and (f), we can know that the third harmonic and fifth harmonic are extracted from the envelope after the second and third atomic decomposition. The method can reproduce the rectangular wave signal with high accuracy.

\section{Conclusions}

In this paper, the spectrum of the envelope of voltage fluctuation signal is analyzed by using the method of atomic decomposition. Using real-coded quantum evolutionary algorithm to optimize the MP matching algorithm, a new method to calculate the $P_{s t}$ of the short time flicker severity in power system is presented. Simulation results show that the proposed method is used to calculate $P_{s t}$ with high accuracy, and that it is very stable. The method based on atomic decomposition and real coded quantum evolutionary algorithm not only provides a new way for the calculation of $P_{s t}$ in power systems, but also provides a tool for solving similar problems in related fields.

\section{Acknowledgements}

This research was supported by the Application Technology Research and Development Projects of Harbin of China (No. 2015RAXXJ036); Heilongjiang Province Science Foundation of China (No. E2015062); Applied Fundamental Research Plan Project of Sichuan Province of China (No. 2015JY0234).

\section{References}

1. A. J. Zhang, X. F. Xiao, X. Fu and D. Wang: "Research on new energy power grid-integration on grid power quality," International Journal of Grid and Distributed computing, Vol.9, No.4, pp.1-12 (2016)

2. H. X. Zhao, M. Z. Chen and H. Z. Dai: "Error analysis of discrete calculation method of flicker severity and its application," Power System Protection and Control, Vol.13, No.28, pp.84-87 (2004)

3. C. F. Sun and H. Gao: "a method to improve calculating accuracy of the short term flicker severity Pst," Power System Protection and Control, Vol.36, No.16, pp.48-51 (2008)

4. H. Wen, Z. S. Teng, F. H. Li, Y. Wang and X. G. Hu: "Improved windowed spectral-peaks polynomial fitting algorithm and its application in power harmonic analysis," Transactions of China Electrotechnical Society, Vol.26, No.10, pp.8-15 (2011)

5. S. Y. Hou, W. Y. Zhang, T. Sun, J. F. Chen and X. W. Zou: "Power quality disturbances analysis using timefrequency atom decomposition optimized by genetic algorithm," Proceedings of the CSEE, Vol.28, No.33, pp.106$113(2013)$

6. Y. P. Gao, X. S. Teng, H. Wen and B. Zeng: "Harmonic analysis based on Kaiser window interpolation FFT and its application," Proceedings of the CSEE, Vol.30, No.4, pp.43-48 (2010)

7. L. Zhang, C. Huang, Y. Q. Jiang, H. M. Yu, M. Lei and J. Mei: "Identification analysis of power quality disturbances based on harmony search and atom decomposition," Power System Technology, Vol.39, No.6, pp.194-201 (2015)

8. A. Y. Wei, Y. J. Guo and X. Z. Cheng: "ECG signal denoising based on improved MP algorithm," International Journal of Signal Processing, Image Processing and Pattern Recognition, Vol.8, No.11, pp.329-338 (2015)

9. S. Yang, S. Y. Cao, C. H. Dai, Y. F. Zhu and W. R. Chen: "Evolutionary matching pursuit based time-frequency atom decomposition for power quality disturbance signals," Power System Protection and Control, Vol.16, No.43, pp.79-86 (2015)

10. M. Li and X. R. Wang: "Detection of voltage flicker based on Time-frequency atom method," Power System Technology, Vol.34, No.7, pp.94-97 (2010)

11. H. Gao, Q. C. Song and J. Huang: "Subgrade settlement prediction based on least square support vector regression and real-coded quantum evolutionary algorithm," International Journal of Grid and Distributed Computing, Vol.9, No.7, pp.83-90 (2011)

12. H. Gao and J Huang: "Combined forecasting model of subgrade settlement based on forecasting availability and realcoded quantum evolutionary algorithm," International Journal of Signal Processing, Image Processing and Pattern Recognition, Vol.8, No.10, pp.147-154 (2016)

13. Sun J, Wang W, Kou L, et al: “A data authentication scheme for UAV ad hoc network communication,"Journal of Supercomputing, No.8, pp.1-16 (2017)

14. Shi C, Dou Z, Lin Y, et al: "Dynamic threshold-setting for RF-powered cognitive radio networks in non-Gaussian noise," Physical Communication, (2018)

15. Lin Y, Wang C, Ma C, et al: "A new combination method for multisensor conflict information," Journal of Supercomputing, Vol.72, No.7, pp. 2874-2890 (2016)

16. Wu Q, Li Y, Lin Y: "The application of nonlocal total variation in image denoising for mobile transmission," 
Multimedia Tools \& Applications, Vol.76, No.16, pp. 1-13 (2016)

17. Lin $Y$, Zhu X, Zheng Z, et al. "The individual identification method of wireless device based on dimensionality reduction and machine learning," Journal of Supercomputing, No 5, pp.1-18(2017)

18. Yun Lin, Chao Wang, Jiaxing Wang, Zheng Dou. "A Novel Dynamic Spectrum Access Framework Based on Reinforcement Learning for Cognitive Radio Sensor Networks”. Sensors, Vol.16, No.10, pp. 1-22(2016)

19. Sun J, Wang W, Kou L, et al. "A data authentication scheme for UAV ad hoc network communication". Journal of Supercomputing, No.8, pp.1-16(2017)

20. Shi C, Dou Z, Lin Y, et al. "Dynamic threshold-setting for RF-powered cognitive radio networks in non-Gaussian noise". Physical Communication, Vol.27, No.4, pp.99-105(2018) 